\title{
Hybrid Relaying Protocol for Joint Power and Subcarrier Allocation for OFDM Based Cognitive Radio Networks
}

\author{
Muthusamy Bhuvaneswari ${ }^{1}$, Shama Srinivasa Rao Madane ${ }^{2}$ \\ ${ }^{1}$ Department of Electronics and Communication Engineering, Indra Ganesan College of Engineering, \\ Trichy, India \\ ${ }^{2}$ Adhi Parasakthi College of Engineering, Kalavai, India \\ Email: bhuvaneswarim0172@rediffmail.com
}

Received 14 March 2016; accepted 5 April 2016; published 23 August 2016

Copyright (C) 2016 by authors and Scientific Research Publishing Inc.

This work is licensed under the Creative Commons Attribution International License (CC BY). http://creativecommons.org/licenses/by/4.0/

(c) () 0 pen Access

\section{Abstract}

This paper aims to avoid the interference imposed by the secondary user on a primary user in Cognitive Radio Network (CRN). In CRN, the interference from secondary user enforced on primary user mainly depends on spectral interval between primary and secondary systems. Moreover, it also depends on the power allocated to the secondary user. In order to avoid interference imposed by secondary user on primary user, a Hybrid Relaying Protocol for Joint Power and Subcarrier Allocation for Orthogonal Frequency Division Multiplexing (OFDM) based Cognitive Radio Networks is proposed. In hybrid relaying protocol, a secondary user uses amplify and forward (AF) protocol and decode and forward (DF) protocol based on the requirement to maximize network throughput. A greedy algorithm is proposed for the selection of relay to get the optimal solution. Moreover, an efficient hybrid power and subcarrier algorithm is used by considering interference constraint imposed by cognitive network to the primary user.

\section{Keywords}

Orthogonal Frequency Division Multiplexing, Interference, Relay, Power, Amplify and Forward, Decode and Forward, Greedy Algorithm

\section{Introduction}

With the rapid development of wireless communications technology, radio spectrum resource is increasingly scarce. A Cognitive Radio Network (CRN) is composed of both the secondary users with CR-enabled radios and 
the primary users whose radios need not be CR-enabled [1]. For CR systems it was important to keep the interference to the Primary User (PU) band within a tolerable range. Since the joint subcarrier and power allocation problem was a non-convex integer problem and a closed form solution was difficult to find a suboptimal algorithm that separated subcarrier and power allocation. Thus a suboptimal subcarrier algorithm that could allocate subcarriers to CR users that not only increase the capacity, but also reduce the interference was introduced to the PU band.

The CRs are equipped in CRNs that can optimize the performance by adapting to network conditions. In this the radio can change its transmission parameters based on the perceived availability of the spectrum bands in its operating environment [2]. Applications of CRNs include intelligent transport systems, Public safety systems, Femtocells, Cooperative networks, Dynamic spectrum access, and Smart grid communications [3]. The power optimization problem in multiple input multiple output OFDM based CR systems is discussed [3]. An optimal power allocation scheme by capitalizing on the relationship between mutual information and minimum mean square error is derived. They have been planning to save transmit power compared to its conventional counterpart. Furthermore, the system achieves higher data rate compared to the Gaussian optimized power due to fewer number of subcarriers being null.

Sensing time and delay, Channel impairments, Wideband sensing, Energy efficiency, Interference, Spectral efficiency, Cooperation and Mobility are the issues of OFDM based CRN [4]. An energy-efficient resource allocation problem which maximizes the cognitive radio link capacity taking into account the availability of the subcarriers and the limits on total interference generated to the Pus is illustrated in [5]. They have considered it as an energy-aware capacity expression by taking into account another factor called subcarrier availability. Optimizing such an expression saves valuable resources such as battery life by selectively allocating power to underutilized subcarriers. Thus due to the complex structure of the optimal solution, three suboptimal schemes, namely, the step-ladder, nulling, and scaling schemes, are used.

Subcarrier power allocation (or power loading) is a common technique to improve the system performance of OFDM systems by optimally allocating transmits power to the different subcarriers [6]. If the channel state information is available at the transmitter, power loading can be used to either optimize error rate, transmission capacity, or transmit power [7]. The first goal is to achieve the maximum energy-efficiency. Unfortunately, the problem is non-convex. Thus we reformulate it into an equivalent convex one and illustrate the equivalent conditions using parametric programming. Second, the problem becomes even more complex because interference constraint must be considered to guarantee the reliable communication of primary users [8] [9].

Bandwidth and power allocation with amplify and forward (AF) or decode and forward (DF) relaying protocol is discussed in [10] to 1) maximize the sum network throughput; 2) minimize the total transmit power of the CR network with considering the fairness of power drain of relay SUs; 3) maximize the energy efficiency of the CR network. They have mentioned the DF relaying protocol that could achieve better performance when the decoding rate constraint was not considered. In contrast, when considering the decoding rate constraint in DF relaying protocol, the hybrid relaying protocol combines AF and DF relaying protocols. A greedy algorithm is developed to solve the joint optimization problem, which has much less computational complexity.

A power and subcarrier allocation for cooperative CR networks in the presence of spectrum sensing errors is designed in [11]. They have derived the mutual interference of primary and secondary networks affecting each other by taking into account spectrum sensing errors. Then, taking into account the interference constraint is imposed by the cognitive network to the primary user and the power budget constraint of cognitive network that will maximize the achievable data rates of secondary users.

The problem of resources allocation in decode and forward (DF) relayed OFDM based cognitive system is discussed in [12]. The dual decomposition technique was adopted to obtain an asymptotically optimal subcarrier pairing, relay selection, and power allocation. The resources were optimized under the individual power constraints in source and relays so that the sum rate was maximized while the interference induced to the primary system was kept below a pre-specified interference temperature limit. Furthermore, a sub-optimal scheme was shown to avoid the high computational complexity of the optimal scheme. The suboptimal algorithm allocated jointly the different resources taking into account the channel qualities, the DF-relaying strategy, the interference induced to the primary system, and the individual power budgets.

The resource allocation problem in multiuser OFDM based CRNs is demonstrated in [8]. The interference to Pus was fully measured, as well as a set of proportional rate constraints to ensure fairness among SUs. Since it was extremely computationally complex to obtain the optimal solution because of integer constraints, they have 
adopted a two-step method to address the formulated problem. Thus a heuristic sub channel assignment was developed based on the normalized capacity of each OFDM sub channel by jointly considering channel gain and the interference to PUs. It was a rough proportional fairness that removed the intractable integer constraints.

The objective of this work is to analyze the effect of interference introduced by the primary user signal to the CR signal and then solve the problem of power and subcarrier allocation in OFDM-CRN. As an extension to [13], we develop a hybrid relaying protocol for joint power and subcarrier allocation. A suboptimal low complexity power and subcarrier allocation algorithm is then proposed considering the interference constraint imposed by the cognitive network to the primary user.

\section{Methods and Materials}

As an extension to this work, we develop a hybrid relaying protocol for bandwidth and power allocation. A suboptimal low complexity power and subcarrier allocation algorithm is then proposed considering the interference constraint imposed by the cognitive network to the primary user. In hybrid relaying protocol [11], a relay SU uses the AF relaying protocolonly if it cannot reliably decode the source data. Otherwise, a relay SU uses the DF relaying protocol. A greedy algorithm is used to select the relay. The effect of interference introduced by the primary user signal to the CR signal is analyzed in terms of the false alarm probability [10]. Then the joint power and subcarrier allocation problem is solved.

Figure 1 represents the proposed block diagram. In our proposed technique, a hybrid relaying protocol is used for efficient allocation of bandwidth and power in the sub-band of CR network. For this SU uses AF relay protocol, only in case it is unable to decode the source data, else it uses the DF relaying protocol. The selection of relay is done using greedy algorithm to achieve optimal solution. After that estimation of interference introduced by primary signal to CR signal. The power allocation is done in order to enhance the possible information rate for the CR network whereas maintaining the instantaneous interference generated to the primary below a pre-defined threshold.

\subsection{Hybrid Relaying Protocol}

Usually it is assumed that relay SU can execute the DF relaying protocol in case the data rate between source

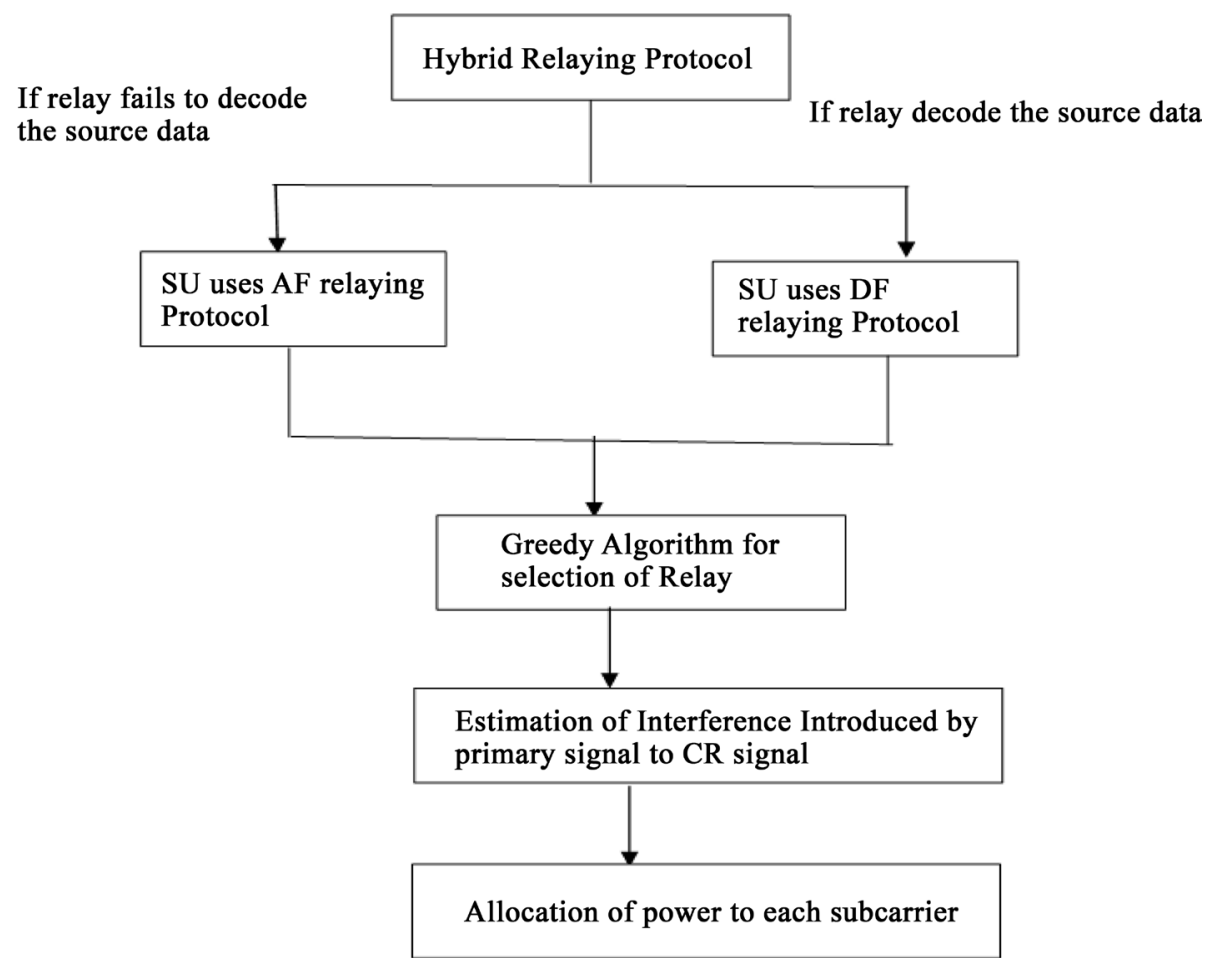

Figure 1. Proposed hybrid relaying protocol. 
SU and relay SU is greater than zero. In this case, the data rate between the source SU and relay SU is greater than $t(t>0)$ to assure reliable decoding in relay SU. This decoding rate limit can make some poor quality of relaying link to acquire more resource. Hence, it will lead to wastage of resources.

This section describes about a hybrid relaying protocol to overcome the above mentioned issue, in which a relay SU make use of AF relaying protocol only in case it cannot reliably decode the source data, else SU uses the DF relaying protocol. Hence, the joint bandwidth and power allocation issue with hybrid relaying protocol can be formulated as below:

$$
\begin{aligned}
& \max _{U_{k}, I_{k}^{R}, I_{k}^{S} v_{k}} \sum_{k=1}^{K}\left[v_{k} S_{k, R C}^{C G}+\left(1-v_{k}\right) S_{k, R C}^{B G}\right] \\
& \text { s.t. } \quad v_{k}\left(t-S_{k, R S}^{C G}\right) \leq 0, k=1,2, \cdots, K \\
& t_{k}-\left[v_{k} S_{k, R C}^{C G}+\left(1-v_{k}\right) S_{k, R C}^{B G}\right] \leq 0 \\
& k=1,2, \cdots, K
\end{aligned}
$$

where $v=\left[v_{1}, v_{2}, \cdots, v_{k}\right]$ is a binary vector which represents the transmission mode of the relay SUs. In case, $v_{k}=1$ (resp. $\left.v_{k}=0\right)$, then it represents that relay SU uses the DF relaying protocol(resp. the AF relaying protocol). $t_{k}$ represents the minimum acceptable throughput for the $k^{\text {th }}$ relaying link. $v$ denotes the relay SU's decoding rate constraint in case the relay SU adopts for DF relaying protocol. It is quite difficult to find the optimal solution for above framed problem. Hence, a greedy algorithm is used to find the optimal solution.

\subsection{Greedy Algorithm}

This section describes about the steps involved in the greedy algorithm [11] to obtain the optimal solution:

Algorithm: The objective is to find the optimal $v_{k}, U_{k}, I_{k}^{R}$ and $I_{k}^{S}$.

Step 1: Initialize

$$
\begin{array}{ll}
\eta_{(0)}=\{1,2, \cdots, K\} & Q_{(0)}=\phi \\
v_{(0)}=[0,0, \cdots, 0] . & S_{(0)}=0
\end{array}
$$

$J=1$

For $j^{\text {th }}$ iteration:

Step 2: if $j>K$, then go to step 4 .

Else

$$
\eta_{j}=\{1,2, \cdots, K\}-Q_{(j-1)}
$$

for each relaying link $k \in \eta_{(j)}$ do

$$
v=v_{(n-1)}, \quad v_{k}=1
$$

Solve the optimization problem for specific $v$ and obtain the sum throughput

$$
S_{(j), k}=S\left(U_{k}, I_{k}^{R}, I_{k}^{S}, V_{k}\right)
$$

if the optimization problem for specific $v$ has no feasible solution, then the throughput $S_{(j), k}=0$.

End for

Step 3: if $\max _{k \in \eta_{(j)}} S_{(j), k} \leq S_{(j-1)}$, go to step 4
Else

$$
\begin{array}{cc}
q_{(j)}=\arg \max S_{(j), k}, & Q_{(j)}=Q_{(j-1)} \cup\left\{q_{(j)}\right\} \\
v_{(j)}=v_{(j-1)}, & v_{q_{(j)}}=1 \\
S_{(j)}=S_{(j)} q_{(j)}, & j=j+1
\end{array}
$$


Go to step 2.

Step 4: $v_{(j-1)}$ is the optimal $v$. Finally, the optimal $U_{k}, I_{k}^{R}$ and $I_{k}^{S}$ can be obtained through solving optimization problem for the optimal $v$.

In the initialization stage, it is assumed that all relay SUs selects AF transmission mode. In the above mentioned greedy algorithm, $\eta_{(j)}$ is represented as candidate set which includes the indices of relay SU that have not selected DF transmission mode and are qualified to select DF transmission mode at the $j^{\text {th }}$ iteration.

$Q_{(j)}$ includes the indices of the relay SUs that have selected DF transmission mode after the $j^{\text {th }}$ iteration. $v_{(j)}$ represents the transmission mode of all relay SU after $j^{\text {th }}$ iteration. $S_{(j)}$ denotes the sum throughput attained on the specific transmission mode $v_{(j)} . q_{(j)}$ denotes the index of the relay SU that selects DF transmission mode at the $j^{\text {th }}$ iteration.

The main aim of adopting greedy algorithm is as follows:

1) For each iteration, all relay SUs that have not selected DF transmission mode can be selected separately.

2) For each of the selected relay $\mathrm{SU}$ at this specific iteration, its AF transmission mode can be changed to DF transmission mode that forms a new specific $v$.

3) By solving the optimization problem for the $v$, the sum throughput is achieved.

4) Relay SU that provides largest sum throughput at this iteration transforms initial transmission mode to DF transmission mode.

5) This algorithm repeats the process for the remaining SUs that have not selected DF transmission mode till all relay SUs have selected DF transmission mode or sum throughput terminates increasing from one-iteration to the next.

\subsection{Effect of Interference Caused by False Alarm Probability}

This section first describes about the effect of interference caused by false alarm probability. Figure 2 represents the availability of total bandwidth primary bandwidth which is available to the CR network. In Figure 2 the total available bandwidth is divided into $\mathrm{Z}$ sub-band with a bandwidth equal to $\Delta f$. Due to lack of perfect spectrum sensing, accurate information about presence of primary signal in each sub-band is not available and, hence CR user can transmit data through each sub-band. For this first we need to define the probability $P\left(\hat{G}_{0}^{i}\right)$ as:

$$
\begin{aligned}
P\left(\hat{G}_{0}^{i}\right) & =P\left(\hat{G}_{0}^{i}, G_{0}^{i}\right)+P\left(\hat{G}_{0}^{i}, G_{1}^{i}\right) \\
& =P\left(\hat{G}_{0}^{i} \mid G_{0}^{i}\right)+P\left(G_{0}^{i}\right)+P\left(\hat{G}_{1}^{i} \mid G_{1}^{i}\right) P\left(G_{1}^{i}\right) \\
& =\left(1-P_{f l}^{i}\right) P\left(G_{0}^{i}\right)+\left(1-P_{e}^{i}\right) P\left(G_{1}^{i}\right)
\end{aligned}
$$

where $P_{f l}^{i}=P\left(\hat{G}_{0}^{i} \mid G_{0}^{i}\right)$ represents the false-alarm probability in the $i^{\text {th }}$ sub-band. $P_{e}^{i}=P\left(\hat{G}_{1}^{i} \mid G_{1}^{i}\right)$ represents the detection probability in the $i^{t h}$ sub-band. Now, by considering Equation (1), the conditional probability $\chi_{i}$ can be derived and defined as below:

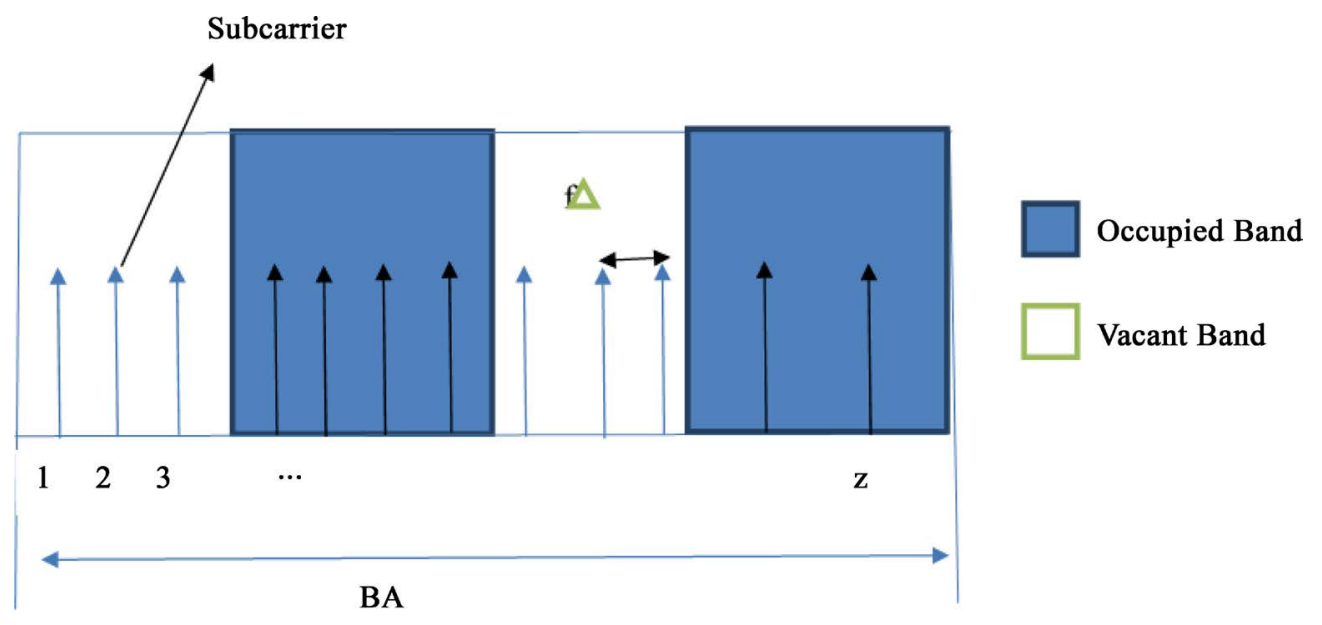

Figure 2. Availability of total primary bandwidth for an opportunistic access to cognitive radio networks. 


$$
\chi_{i} \triangleq P\left(G_{0}^{i} \mid \hat{G}_{0}^{i}\right)=\frac{P\left(G_{0}^{i} \mid \hat{G}_{0}^{i}\right)}{P\left(\hat{G}_{0}^{i}\right)}=\frac{P\left(G_{0}^{i} \mid \hat{G}_{0}^{i}\right) P\left(G_{0}^{i}\right)}{P\left(\hat{G}_{0}^{i}\right)}=\frac{\left(1-P_{f l}^{i}\right) P\left(G_{0}^{i}\right)}{P\left(\hat{G}_{0}^{i}\right)}
$$

\subsection{Estimation of Interference Introduced by Primary's Signal to CR's Signal}

In this section, we will estimate the interference produced by primary's signal to the CR's signal. The power density spectrum of the primary signal after N-point fast Fourier transform (FFT), can be presented by the estimated value of the periodogram as below:

$$
F\left\{Y_{N}(u)\right\}=\frac{1}{2 \pi N} \int_{-\pi}^{\pi} \phi P W\left(b^{q u}\right)\left(\frac{\sin (u-\lambda) N / 2}{\sin (u-\lambda) / 2}\right)^{2} \mathrm{~d} \lambda
$$

where $u$ represents the frequency normalized to the sampling frequency. $\phi P W\left(b^{q u}\right)$ denotes the power density spectrum of the primary goal. The interference generated by the $m^{\text {th }}$ primary sub-carrier to the $i^{\text {th }} \mathrm{CR}$ sub-carrier which is allocated to the $k^{\text {th }}$ secondary user, represented as $O_{k, m i}$ can be represented as below:

$$
O_{k, m i}=\left|x_{k, m}^{C D}\right|^{2} \int_{\left(d-\frac{1}{2}\right) \Delta f}^{\left(d+\frac{1}{2}\right) \Delta f} F\left\{Y_{N}(u)\right\} \mathrm{d} u
$$

where $d=|m-i|$, i.e., $d \Delta f$ represents the distance in frequency between the $i^{\text {th }}$ CR subcarrier and the $m^{\text {th }}$ primary subcarrier. The main goal is to derive the interference introduced by the $m^{\text {th }}$ primary subcarrier to the $i^{\text {th }}$ $\mathrm{CR}$ subcarrier under the consideration that the spectrum sensing process is imperfect. In other words, it can be said that, the primary and the CR both can potentially broadcasts simultaneously over the same sub-carrier, which means, in case the spectrum sensing process make inaccurate decision, then there exists interference scenario where $i=m$.

It is assumed that the spectrum sensing process has made its decision in favor of one of the two hypothesis $\hat{G}_{0}^{i}$ (i.e., the primary is operation in $i^{\text {th }}$ sub-band) or $\hat{G}_{1}^{i}$ (i.e., the primary operation in $i^{\text {th }}$ sub-band. However, based on the spectrum sensing decision $\left(\hat{G}_{0}^{i}\right.$ or $\hat{G}_{1}^{i}$ ), the interference level is different. The main goal of this part is to derive and differentiate these interference levels under an imperfect spectrum sensing.

If the decision of spectrum sensing block is $\hat{G}_{0}^{i}$, then the secondary network can broadcast data in the $i^{\text {th }}$ sub-band. Whereas, if the decision is $\hat{G}_{1}^{i}$, then the secondary network will not transmit any data in the $i^{\text {th }}$ sub-band. If the decision of spectrum sensing block is $\hat{G}_{0}^{i}$, then one of the following two cases occurs:

1 ) The primary is not present in the $i^{\text {th }}$ sub-band $\hat{G}_{1}^{i}$. Hence, both primary and secondary will transmit data simultaneously on this sub-band and the wrong decision of spectrum generates intense interference. Moreover, it is significant to consider the primary interference in other sub-band on the secondary in $i^{\text {th }}$ sub-band.

2) In second case, the primary is not present in $i^{\text {th }}$ sub-band $\hat{G}_{1}^{i}$. The spectrum sensing block takes the right decision and there exist only primary interference in other sub-band on the secondary in the $i^{\text {th }}$ sub-band.

Hence, the average total interference forced by the primary network on the $k^{\text {th }}$ secondary operating in $i^{\text {th }}$ sub-band under imperfect spectrum sensing, represented $\tilde{O}_{k, i}$, writes:

$$
\begin{aligned}
\tilde{O}_{k, i} & =\sum_{m=1}^{Z} P\left(G_{1}^{m}, \hat{G}_{0}^{i}\right) O_{k, m i} \\
& =\sum_{m=1, m \neq i}^{Z} P\left(G_{1}^{m}, \hat{G}_{0}^{i}\right) O_{k, m i}+P\left(G_{1}^{i}, \hat{G}_{0}^{i}\right) O_{k, i i} \\
& =\sum_{m=1, m \neq i}^{Z} P\left(G_{1}^{m}\right) P\left(\hat{G}_{0}^{i}\right) O_{k, m i}+P\left(G_{0}^{i} \mid \hat{G}_{0}^{i}\right) P\left(\hat{G}_{0}^{i}\right) O_{k, i i} \\
& =P\left(\hat{G}_{0}^{i}\right)\left[\sum_{m=1, m \neq i}^{Z} P\left(G_{1}^{m}\right) O_{k, m i}+\left(1-\chi_{i}\right) O_{k, i i}\right]
\end{aligned}
$$

where $O_{k_{i} i i}$ represents the interference caused by simultaneous transmission of the primary and $k^{\text {th }}$ secondary over the $i^{i t h}$ sub-carrier. 


\subsection{Hybrid Power and Subcarrier Allocation Technique}

The main goal is to allocate power to each subcarrier of the OFDM based CR in order to enhance the possible information rate for the CR network whereas maintaining the instantaneous interference generated to the primary below a pre-defined threshold.

Consider, an ideal coding scheme which uses the Shannon Capacity formula, then the cognitive attainable data rate at the $i^{\text {th }}$ subcarrier which is assigned to the $k^{\text {th }}$ secondary, represented as $X_{k, i}$ for $i \in\{1, \cdots, Z\}$ and $k \in\{1, \cdots, K\}$,

$$
X_{k, i}\left(p_{k, i}\right)=\chi_{i} \Delta f \log _{2}\left[1+\frac{\left|e_{k, i}^{H H}\right| p_{k, i}}{\omega_{k, i}^{2}+\tilde{O}_{k, i}}\right]
$$

where $p_{k, i}$ represents total transmit power in the $i^{\text {th }}$ subcarrier which is assigned to the $k^{\text {th }}$ secondary, and $\omega_{k, i}^{2}$ denotes the additive white Gaussian noise (AWGN) variance.

By considering the Equation (10), hybrid cognitive power allocation under imperfect spectrum sensing is expressed as the following optimization problem:

$$
V=\max _{p_{k, i}, y_{k, i}} \sum_{k=1}^{K} \sum_{i=1}^{Z} y_{k, i} X_{k, i}\left(p_{k, i}\right)
$$

Subject to:

$$
\begin{gathered}
\sum_{k=1}^{K} \sum_{i=1}^{Z} y_{k, i}, \bar{F} \leq F_{t h}, \\
\sum_{k=1}^{K} \sum_{i=1}^{Z} y_{k, i}, p_{k, i} \leq O, \\
\sum_{k=1}^{K} y_{k, i} \leq 1 \\
p_{k, i} \geq 0, \quad \forall k \forall i \\
y_{k, i} \in\{0,1\}, \quad \forall k \forall i
\end{gathered}
$$

where $V$ denotes the transmission capacity of the CR user, $F_{t h}$ represents the total tolerable interference at primary and $O$ represents the maximum power constraint at the CR network. $y_{k, i}$ is basically a binary variable representing that subcarrier $\mathrm{i}$ is assigned to secondary user $k$ or not, while each subcarrier can be assigned to only one secondary user. The optimization problem represented in eq. Sohail, A., et al (2013) for $K=1$ is a standard convex problem that can be solved easily with the help of Lagrange multiplier method. The overall algorithm is given below:

1) //Hybrid Relaying protocol//,

2) If SU can decode the data source,

3) Then use AF relaying protocol,

4) Else, it use DF relaying protocol,

5) Use Greedy Algorithm for relay selection,

6) Compute the effect of interference caused by false alarm probability,

7) Estimate the interference introduced by primary's signal to CR's signal,

8) Allocate power to each sub-carrier of the OFDM.

\section{Results and Discussion}

The simulation results and discussion shown below gives the efficiency of our proposed work. The simulation is done using Matlab7.12 (R2011a). The parameters used for our work is shown in the Table 1 below.

Here for simulation purpose, the user specifies the allocated band for the primary user so if you see the output below we have 3 primary user (1, 2 and 5) rest are free band (3 and 4) from which the secondary users can occupy but in the spectrum sensing method of Periodogram. It has given the wrong decision. Spectrum is assigned 
to secondary user but the user 1 is using by the primary user by this the interference is created. Figure 3 shows the command window of the proposed system.

The primary user1, primary user2 and primary user5 are present as shown in Figure 4 and if the secondary

Table 1. Parameters used for our simulation.

\begin{tabular}{cc}
\hline Parameters & Values \\
\hline FFT Size & 64 \\
Number of Carriers in OFDM symbol & 52 \\
Channel & AWGN \\
rk & 1 \\
SNR & $1: 2: 20$ \\
\hline
\end{tabular}

Do you want to enter 1st primary user $\mathrm{Y} / \mathrm{N}$ : $\quad \mathrm{Y}$ Do you want to enter 2nd primary user $\mathrm{Y} / \mathrm{N}$ : $\mathrm{Y}$ Do you want to enter 3rd primary user $Y / N$ : $n$ Do you want to enter 4th primary user $\mathrm{Y} / \mathrm{N}$ : $\quad$ n Do you want to enter 5 th primary user $\mathrm{Y} / \mathrm{N}$ : $n$

Do you want to enter a secondary user $\mathrm{Y} / \mathrm{N}$ : $\mathrm{Y}$ spectrum is assigned to User 1 as it was not present.

$f_{\underline{x}} k \gg 1$

Figure 3. Output window.

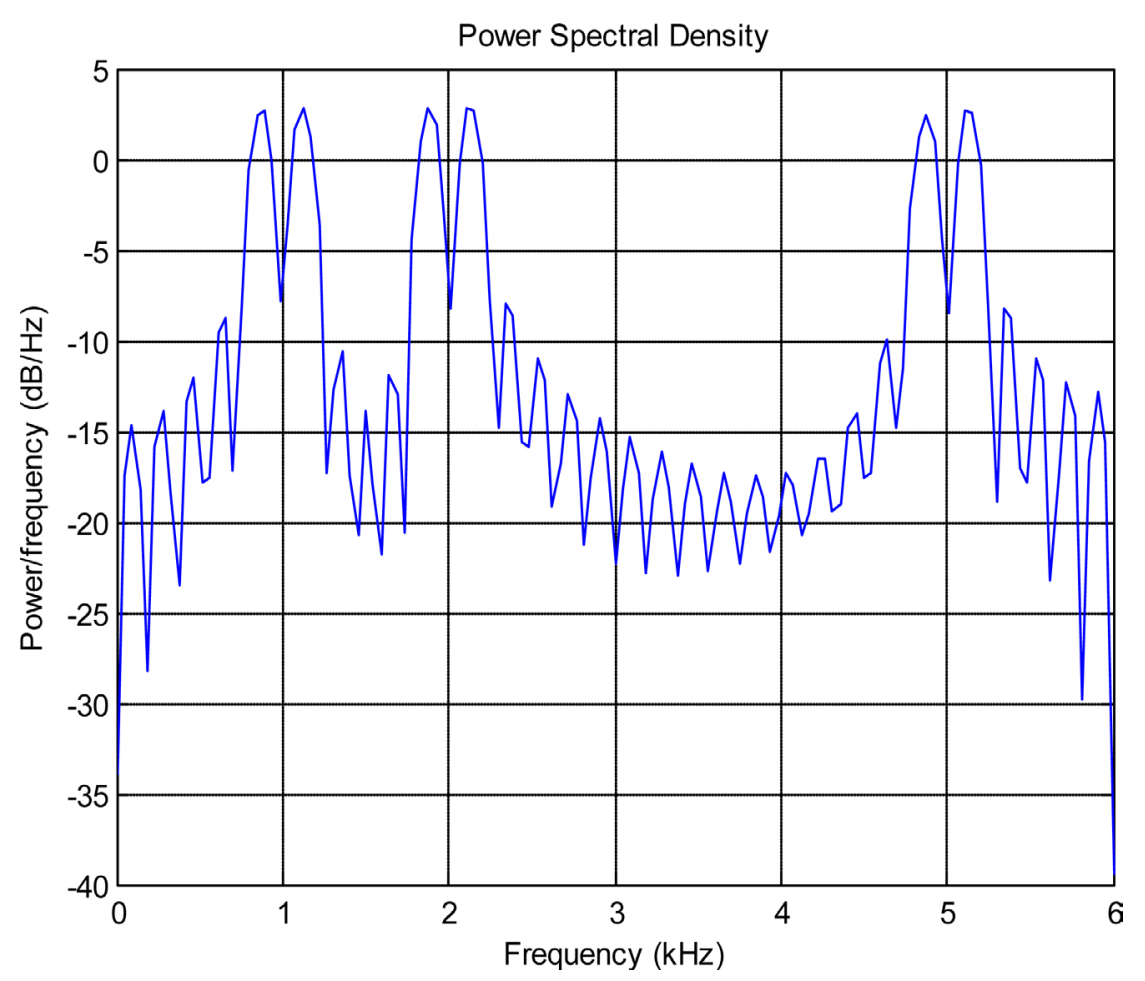

Figure 4. Power/frequency vs. frequency for primary user 1, primary user 2, and primary user 5. 
user wants to transmit the data, then sensing of spectrum occurs in which it finds the primary user 1 is not using the band as wrong decision and that particular band is allocated to that secondary user as shown in Figure 5.

Figure 6 depicts the maximum transmitted data rate of CR user versus the average interference imposed to the primary user band in which the transmit power budget is fixed and set to $3 \mathrm{Watt}$. This figure shows that the proposed scheme leads to higher CR data rates for a given interference level imposed to the primary user band.

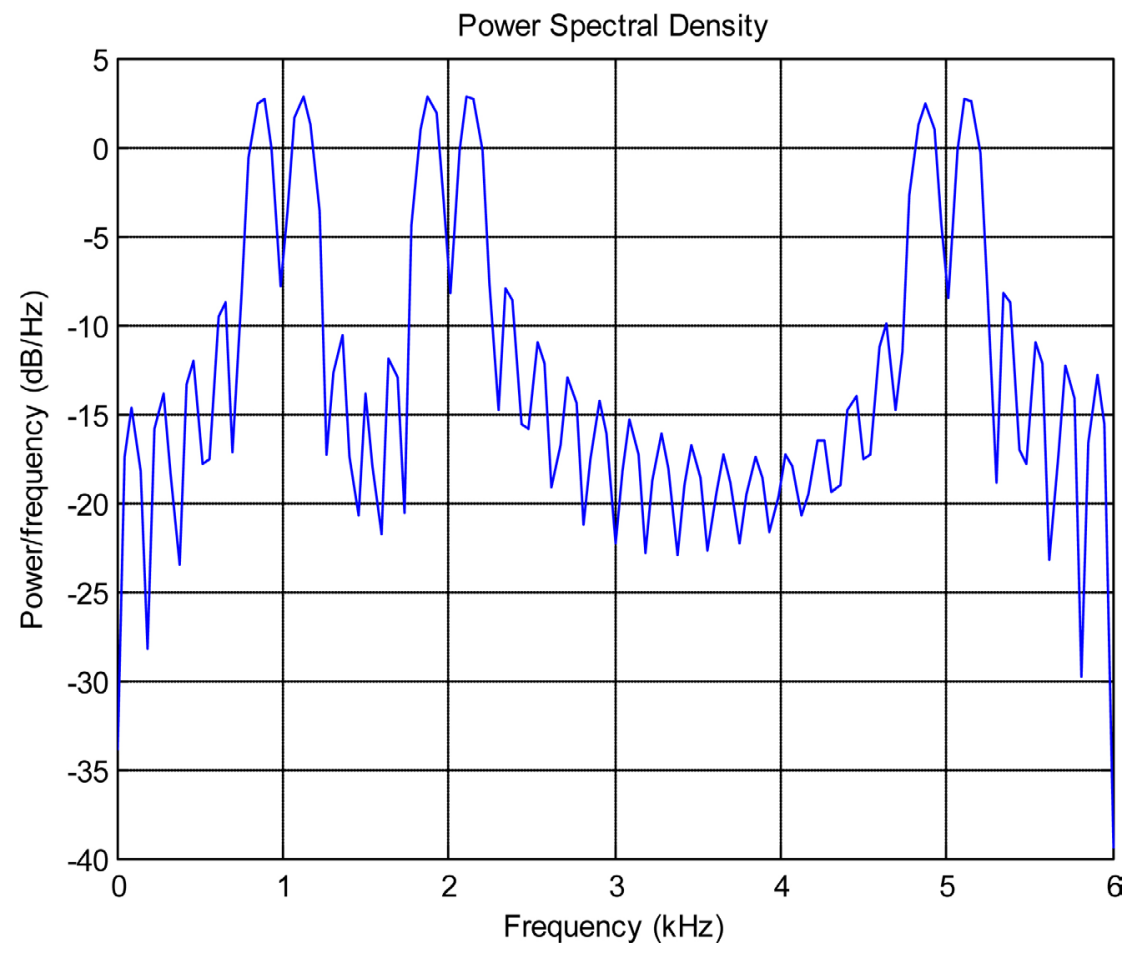

Figure 5. Power/frequency vs. frequency for primary user1as well as secondary user 1, primary user 2 and primary user 4.

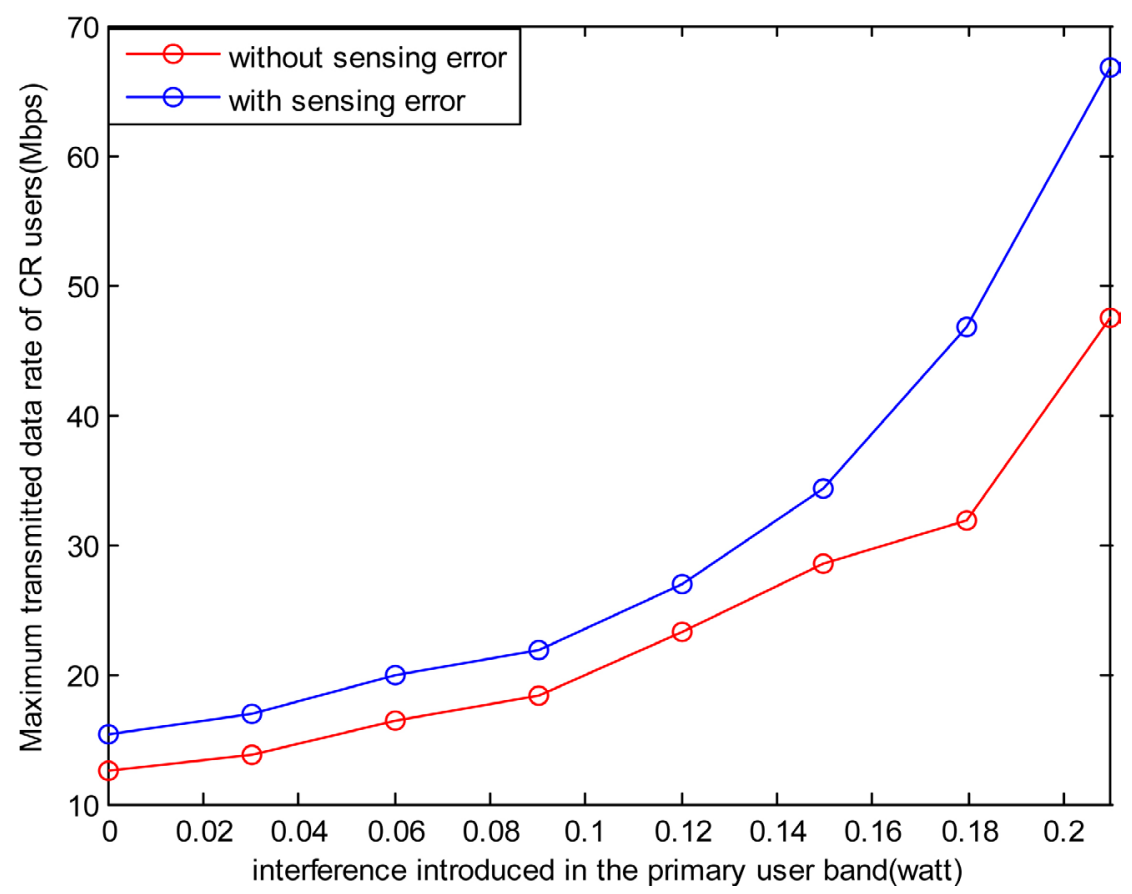

Figure 6. Maximum achieved data rate of CR user versus the average interference imposed to the primary user band. 
Figure 7 gives the power allocation by associate waterfilling approach. It is observed that power allocation by associate waterfilling approach is better than the power allocation by conventional waterfilling because the reduction of error rate is higher for associate waterfilling method.

In Figure 8 and Figure 9, we have introduced the plot for the proposed work power allocation by hybrid relay using greedy algorithm. In both the graph our proposed work outperforms well than the existing method the data rate is higher for the proposed work which gives 91.9 Mbps of data rate as well as in the error rate the reduction of error is higher for the power allocation (hybrid relay using greedy algorithm) the error rate is reduced up to $10^{-4.6}$ at $20 \mathrm{~dB}$.

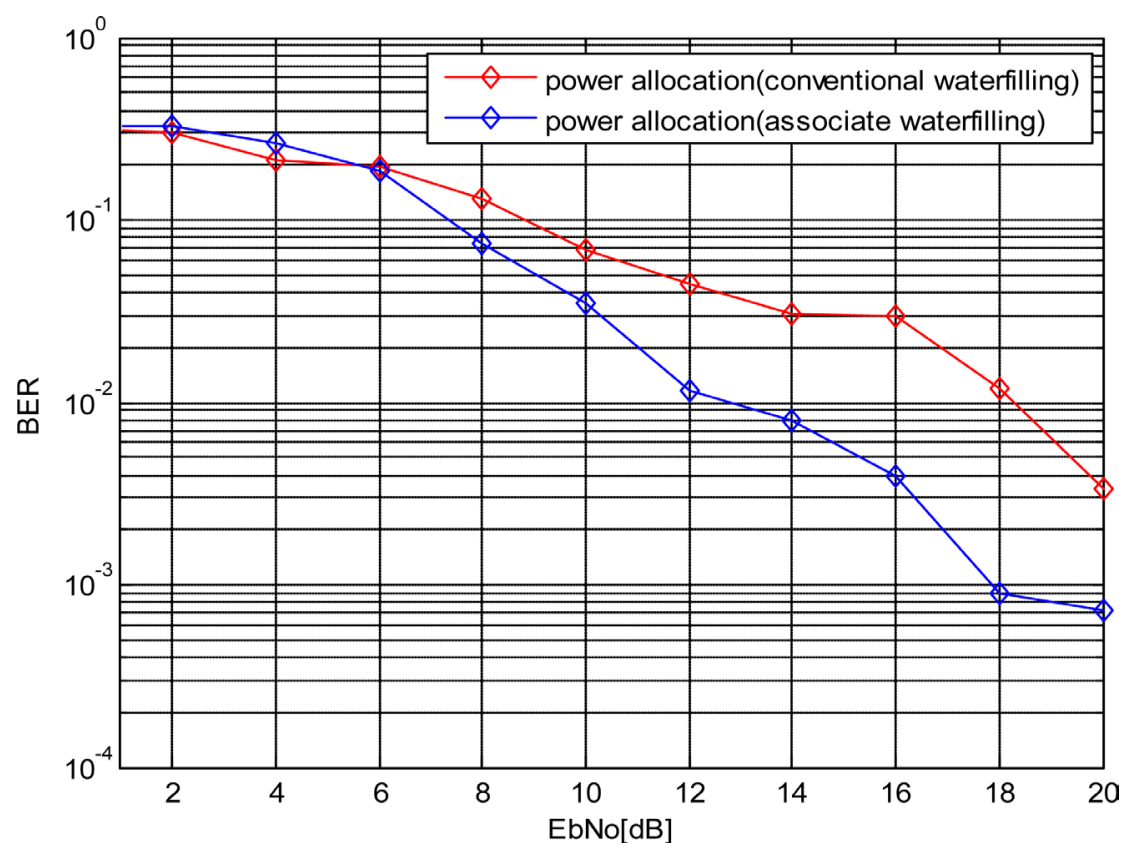

Figure 7. BER vs. SNR for power allocation methods by conventional and associate waterfilling.

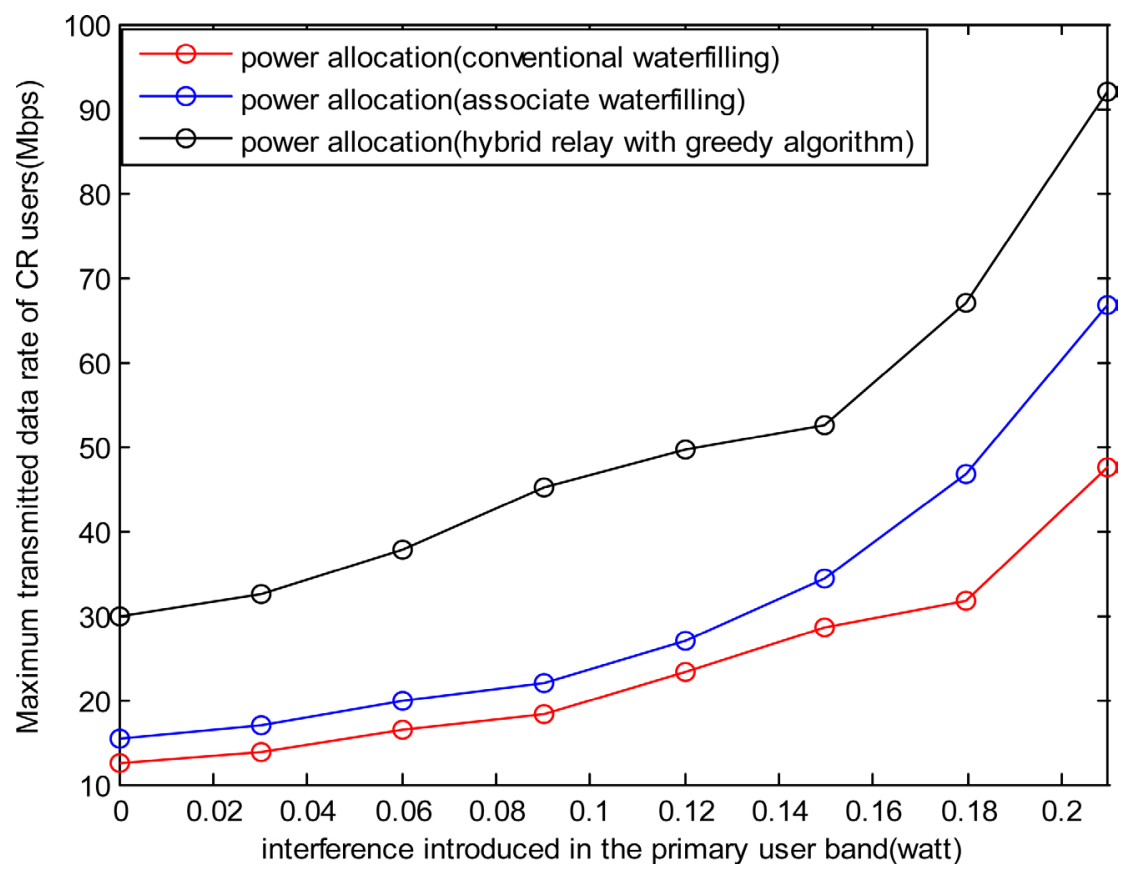

Figure 8. Maximum achieved data rate of CR user vs. the average inference imposed to the primary user band. 


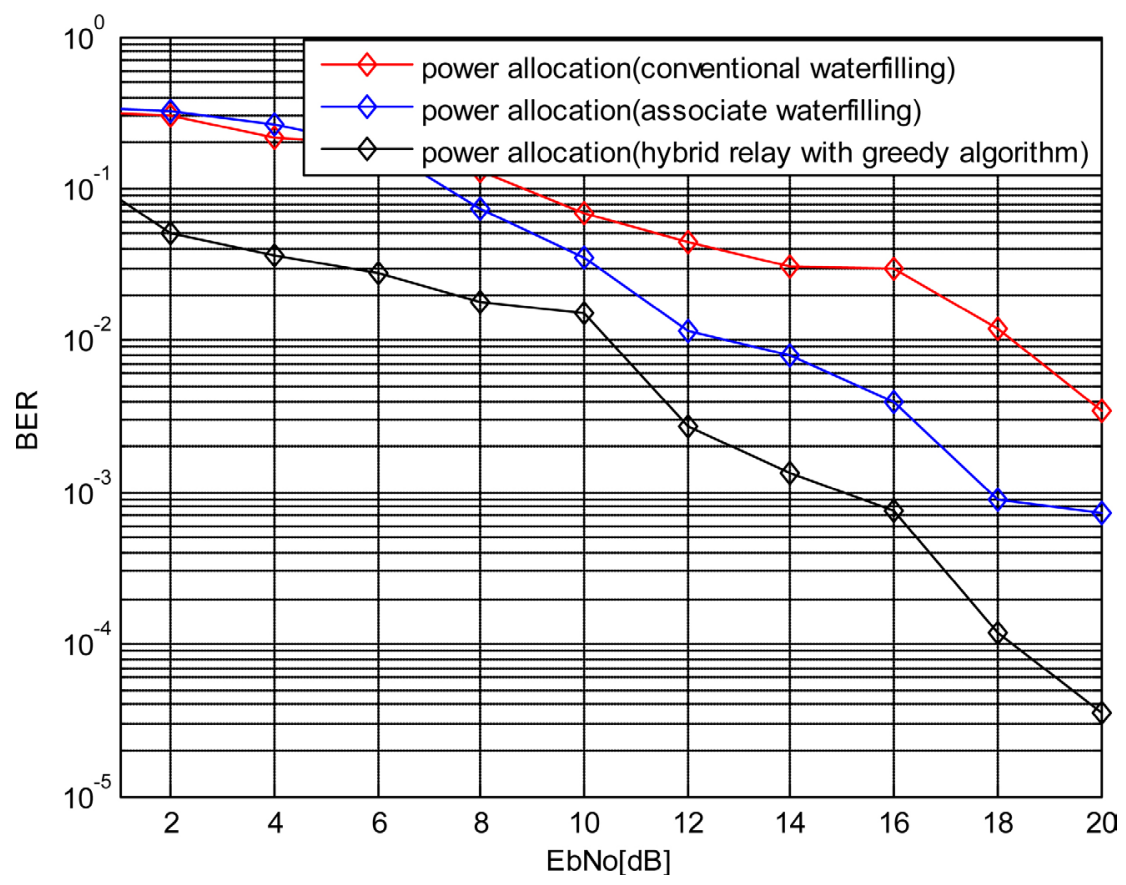

Figure 9. BER Vs SNR for power allocation methods by conventional, associate and hybrid relay with greedy algorithm.

\section{Conclusion}

In this paper, we have proposed a Hybrid Relaying Protocol for Joint Power and Subcarrier Allocation for OFDM based Cognitive Radio Networks. In hybrid relaying protocol, a secondary user uses amplify and forward (AF) protocol and decode and amplify (DF) protocol based on the requirement. SU uses AF, only in case it is unable to reliably decode the source data and hence it does not exceed the decoding rate threshold. Hence, it uses the resource efficiently and maintains the quality of the relaying link. A greedy algorithm is proposed for the selection of relay to get the optimal solution. Moreover, an efficient hybrid power and subcarrier algorithm is used by considering interference constraint imposed by cognitive network to the primary user. Hence, we achieve to maintain the interference below the pre-defined threshold.

\section{References}

[1] Bansal, G., Hasan, Z., Hossain, M.J. and Bhargava, V.K. (2010) Subcarrier and Power Adaptation for Multiuser OFDM-Based Cognitive Radio Systems. IEEE National Conference on Communications, Chennai, 29-31 January 2010, 1-5. http://dx.doi.org/10.1109/ncc.2010.5430230

[2] Zhang, P., Yang, L.X. and Liu, X. (2010) Subcarrier and Power Allocation in OFDM-Based Cognitive Radio Systems. International Journal of Computer Network and Information Security, 2, 24-30. http://dx.doi.org/10.5815/ijcnis.2010.01.04

[3] Sohail, A., Al-Imari, M., Xiao, P. and Evans, B.G. (2013) Optimal Power Allocation for MIMO-OFDM Based Cognitive Radio Systems with Arbitrary Input Distributions. Proceedings of IEEE 24th International Symposium on Personal, Indoor and Mobile Radio Communications, London, 8-11 September 2013, 1909-1913.

[4] Van Vinh, N. and Shouyi, Y. (2014) Power Allocation Algorithm in OFDM-Based Cognitive Radio Systems. 2014 International Conference on Computing, Management and Telecommunications, Da Nang, 27-29 April 2014, 13-18.

[5] Hasan, Z., Bansal, G., Hossain, E. and Bhargava, V.K. (2009) Energy-Efficient Power Allocation in OFDM-Based Cognitive Radio Systems: A Risk-Return Model. IEEE Transactions on Wireless Communications, 8, 6078-6088. http://dx.doi.org/10.1109/twc.2009.12.090394

[6] Wang, S., Huang, F. and Wang, C. (2012) Adaptive Proportional Fairness Resource Allocation for OFDM-Based Cognitive Radio Networks. Wireless Network, 19, 273-284.

[7] Kang, X., Garg, H.K., Liang, Y.C. and Zhang, R. (2010) Optimal Power Allocation for OFDM-Based Cognitive Radio with New Primary Transmission Protection Criteria. IEEE Transactions on Wireless Communications, 9, $2066-2075$. http://dx.doi.org/10.1109/twc.2010.06.090912 
[8] Wang, Y., Xu, W., Yang, K. and Lin, J. (2012) Optimal Energy-Efficient Power Allocation for OFDM-Based Cognitive Radio Networks. IEEE Communications Letters, 16, 1420-1423. http://dx.doi.org/10.1109/lcomm.2012.070512.120662

[9] Ekin, S., Abdallah, M.M., Qaraqe, K.A. and Serpedin, E. (2012) Random Subcarrier Allocation in OFDM-Based Cognitive Radio Networks. IEEE Transactions on Signal Processing, 60, 4758-4774. http://dx.doi.org/10.1109/TSP.2012.2203126

[10] Yu, H., Tang, W. and Li, S. (2012) Bandwidth and Power Allocation for Cooperative Relay in Cognitive Radio Networks. EURASIP Journal on Advances in Signal Processing, 1, 1-10. http://dx.doi.org/10.1186/1687-6180-2012-224

[11] Fathi, H. and Sadough, S.M.S.S. (2013) Robust Power and Subcarrier Allocation for OFDM-Based Cognitive Radio Networks Considering Spectrum Sensing Uncertainties. Radio Engineering, 22, 810-817.

[12] Shaat, M. and Bader, F. (2011) Asymptotically Optimal Resource Allocation in OFDM-Based Cognitive Networks with Multiple Relays. IEEE Transactions on Wireless Communications, 11, 892-897.

[13] Bhuvaneswari, M. and Madane, S.S.R. (2015) Power Allocation for OFDM Based AF Cooperative Diversity Systems Using Water Filling Algorithm in CRN. International Journal of Applied Engineering Research, 10, 4627-4644.

\section{Submit or recommend next manuscript to SCIRP and we will provide best service for you:}

Accepting pre-submission inquiries through Email, Facebook, LinkedIn, Twitter, etc.

A wide selection of journals (inclusive of 9 subjects, more than 200 journals)

Providing 24-hour high-quality service

User-friendly online submission system

Fair and swift peer-review system

Efficient typesetting and proofreading procedure

Display of the result of downloads and visits, as well as the number of cited articles

Maximum dissemination of your research work

Submit your manuscript at: http://papersubmission.scirp.org/ 